\title{
Simultaneous Cochlear and Auditory Brainstem Implantation in Children With Severe Inner Ear Malformations: Initial Surgical and Audiological Results
}

\author{
*Levent Sennaroğlu, †Mehmet Yaralı, †Gonca Sennaroğlu, †Betül Çiçek Çınar, \\ $\dagger$ Merve Özbal Batuk, †Esra Yücel, †Burçak Bilginer, *Münir Demir Bajin, \\ $\S \|$ Margaret Winter, and 9 Eric P. Wilkinson
}

*Department of ENT; †Department of Audiology; †Department of Neurosurgery, Hacettepe University, Ankara, Turkey; $\S$ Keck School of Medicine of USC; //California State University Los Angeles; and $\mathbf{1 H o u s e}$ Clinic, Los Angeles, CA, USA

Objective: To report the initial surgical and audiological outcomes of three pediatric patients with severe inner ear malformations who were simultaneously implanted with cochlear and brainstem implants in the same surgical session.

Study Design: Retrospective case review.

Setting: Tertiary referral center.

Patients: Three pediatric patients with severe inner ear malformations between ages of 1.9 to 2.5 years, who were simultaneously implanted with cochlear implant in one ear and auditory brainstem implant in the other ear.

Intervention(s): Simultaneous application of cochlar implant in one ear, auditory brainstem implant in the other ear.

Main Outcome Measures: Free field thresholds with cochlear and brainstem implants. Surgical issues are also discussed.
Results: The study is descriptive in nature. Free field thresholds with each device alone and together showed good progress. One of the patients had slower progress possibly due to comorbid CHARGE syndrome.

Conclusions: The results showed good progress in terms of audition with both devices. Simultaneous cochlear and brainstem application serves as a remedy for pediatric patients who are candidates for cochlear implant on one side and brainstem implant on the other side. With this simultaneous application precious time for auditory development is not lost. Key Words: Auditory brainstem implantsCochlear implants-Severe inner ear malformationsSimultaneous application-Surgical and audiological results.

Otol Neurotol 41:625-630, 2020.
Management of pediatric patients with cochlear nerve deficiency (CND) continues to present a dilemma. Main reason is the frequent unsatisfactory outcome with hearing aids and cochlear implants (CI). Although CI may be a salvage treatment when compared with conventional hearing aids, the outcomes of CI in this group show wide variability in results of speech perception and audition.

CI users with CND gain some basic sound detection abilities, but a relatively small percentage gain higher level processes. Young et al. (1) reported that in $10 \mathrm{CI}$ users with $\mathrm{CN}$ hypoplasia or aplasia, only three could achieve open set speech discrimination; yet another three had little or no sound detection abilities. Similarly, Vincenti et al. (2) showed that all of their five pediatric

Address correspondence and reprint requests to Mehmet Yaral, Ph.D., Hacettepe University, Department of Audiology, Faculty of Health Sciences, Sihhiye/Ankara, Turkey; E-mail: mhmtyrl@yahoo. com.tr

The authors disclose no conflicts of interest.

Supplemental digital content is available in the text.

DOI: $10.1097 /$ MAO.0000000000002595
CI users with $\mathrm{CN}$ deficiency improved their speech perception abilities, but only one achieved spoken language. Zhang et al. (3) reported outcomes of nine pediatric $\mathrm{CI}$ users with $\mathrm{CN}$ deficiency indicating that none of them could develop adequate speech perception or speech intelligibility after at least 1 year of follow-up.

In terms of aided thresholds, outcomes of patients with $\mathrm{CN}$ deficiency were found to be poorer compared with other inner ear malformations (IEM). Buchman et al. (4) showed that among CI users with IEMs, CND group had lower pure-tone average levels and lower percentage of open set speech discrimination.

One option to overcome this problem of suboptimal development in speech recognition in CND is bilateral implantation. Sequential bilateral CI in CND showed better aided thresholds in two cases without a related outcome in speech recognition in one of them (5).

Another option in case of suboptimal progress may be removal of the $\mathrm{CI}$ electrode and auditory brainstem implantation (ABI) on the ipsilateral side. Colletti et al. (6) presented outcomes of 21 children with ABI, who had previously a $\mathrm{CI}$ in other centers diagnosed with 
possible CND (absent or hypoplastic). After ABI on the same side, CAP scores of the users were reported to be significantly better.

An option for management without waiting to see the outcomes with CI is initial ABI application. In 2016, we reported the outcome of $\mathrm{ABI}$ in 35 pediatric users with various severe inner ear malformations, who had at least 1 -year device use. Majority of the ABI users could understand common phrases without the aid of visual cues (7). In terms of speech recognition abilities, 29 of 35 could develop closed set speech recognition and open set speech discrimination more than $50 \%$ was achieved by 12 of 35. In terms of CND with hypoplasia, which is the topic of the current work, presence of a cochleovestibular nerve $(\mathrm{CVN})$ resulted in improved aided thresholds than cases with CVN aplasia. Although speech perception abilities develop after ABI, FAPCI scores were found to be lower in $\mathrm{ABI}$ users than average CI users. In fact, pediatric $A B I$ users were shown to be gaining sound recognition and discrimination at 3 months follow-up, but higher level abilities were related to individual factors such as comorbid disorders (8).

Determining the best stimulation option for children with CND is a challenge. Colletti et al. (9) compared the outcomes of two groups of children with CND using CI or ABI. During the follow-up period of 5 years, CAP scores of ABI users were found to be higher (CAP 2-7) than CI users (CAP 0-3). Moreover, CAP scores showed a continuous increment after 2 years among ABI users, but for CI users' scores stabilized at 2 to 4 -year period and did not improve. In addition, two patients could only respond to sounds without identification. In relation to this suboptimal progress, CI devices of five users were removed and $\mathrm{ABI}$ implanted on the same side.

All these findings show that CI application carries a high risk of suboptimal progress in the follow-up years, though it is difficult to assess who is a better candidate for $\mathrm{CI}$ or ABI in the initial evaluation. During CI follow-up, precious time for development of auditory abilities may be lost. Importance of bilateral stimulation was stressed in the Second Consensus Meeting of ABI in complex inner ear malformations (10). As a result, one potential option for this group is the application of CI first, and later $\mathrm{ABI}$ to contralateral side within 1 year if progress is not seen with CI. In fact, our group reported our results in 12th European Symposium on Pediatric Cochlear Implants that six cases who had a CI first and an ABI later, demonstrated a considerable increase in CAP and SIR scores (11). Unfortunately, the duration between the two surgeries is between 1.5 and 2 years. The reason for this delay is improvement at the beginning but reaching a plateau. By the time a decision for an ABI is made, the child is frequently older than 2.5 to 3 years of age. Beginning auditory stimulation at this older age results in diminished incremental benefit of ABI.

Because of these concerns, since 2015 we have started to provide the families the option of simultaneous CI and $\mathrm{ABI}$ surgery, one on each side, in cases of CND with extremely hypoplastic cochlear nerve where expectations from CI are low. This procedure has been suggested to 12 families and performed in six patients until 2019. Only the first three patients with at least 2 years of device use are presented here. Although simultaneous application brings some issues such as length of anesthesia and experience in pediatric neurotologic surgery, providing immediate bilateral hearing stimulation in this difficult group of patients is the main reason for this procedure.

In this article, we report the outcomes of the first three pediatric patients with simultaneous $\mathrm{CI}$ and $\mathrm{ABI}$ surgery in this patient group. Adequate language acquisition was foreseen to be suboptimal if applied with only CI due to radiological finding of CND with nerve hypoplasia/aplasia. This was the main motivation to develop this new algorithm of simultaneous application.

\section{METHODS}

Three pediatric patients with severe inner malformations were simultaneously implanted with $\mathrm{CI}$ in one ear and $\mathrm{ABI}$ in the contralateral ear based on radiological and audiological examinations. All patients were referred to our clinic for ABI procedure and our team evaluated computed tomography (CT) and magnetic resonance imaging (MRI) images for the possibility of CI, at least on one side. If there is an extremely hypoplastic cochlear nerve on one side with slight response with insert ear phones during audiological evaluation, CI procedure was offered to the family besides ABI intervention. The families were informed about the possible risks associated with ABI surgery. Preop audiological tests consisted of insert ear phone testing, auditory brainstem response testing (ABR), and tympanometry. Insert ear phone testing was conducted through the GSI Audera-61 audiometer (Grason Stadler Inc., $\mathrm{MN}$ ), ABR testing through the Vivasonic Integrity System (Vivasonic Inc., Toronto, Canada), and tympanometry through a GSI Tympstar (Grason Stadler Inc., MN). Postop testing included free field audiometric test with pure tones 500 to $4000 \mathrm{~Hz}$ and/or speech sounds / ba/, /sh/ and patients name. Two of the patients were referred to our center from abroad and one patient was diagnosed in our clinic.

Cochlear Nerve Deficiency can be accepted when there is a separate $\mathrm{CN}$ but the size is less than the contralater normal $\mathrm{CN}$ or ipsilateral normal facial nerve (12). In a situation like this, a cochlear implantation is always the choice. However, there is a subgroup of patients where the $\mathrm{CN}$ is hardly visible. Supplemental Content 1, http://links.lww.com/MAO/A937, shows Anormal $\mathrm{CN}$, B-Hypoplastic $\mathrm{CN}$, and $\mathrm{C}$-severe cochlear nerve deficiency (hardly visible $\mathrm{CN}$ ). In addition, in common cavity there is no isolated $\mathrm{CN}$; a common cochleovestibular nerve enters the common cavity. In cases where CVN is thinner than ipsilateral facial nerve (FN), CVN deficiency can be diagnosed. Audiological findings and surgical observations from patient records for each case are given below.

\section{Case 1}

Preop insert ear phone testing showed that 2-year-old female patient had responses to low frequency sounds 250 and $500 \mathrm{~Hz}$ on the right ear (Fig. 1). Normal tympanometric findings were observed. She had comorbid disorder of moderate CHARGE syndrome.

High Resolution Computerised Tomography (HRCT) findings showed that she had bilateral cochlear hypoplasia Type III with cochlear aperture stenosis. On MRI there was severe 


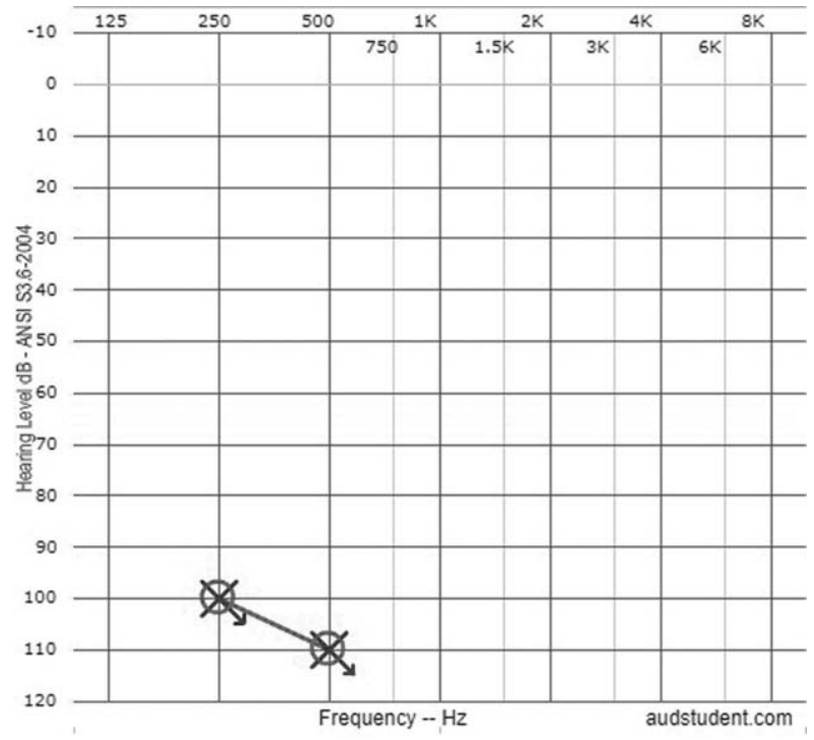

FIG. 1. Insert ear phone test results. No response was observed on left ear.

cochlear nerve deficiency bilaterally where $\mathrm{CN}$ was hardly visible (Fig. 2). As she had clear responses to insert ear phone testing on right ear, CI was planned to right, ABI to left ear. The family was informed about the procedure and in May 2015 simultaneous $\mathrm{CI}$ and $\mathrm{ABI}$ surgery was performed when she was 2 years 2 months.

During surgery for CI, severe FN abnormality was observed as it was found to be overlying the oval and round window and a part of the promontory. Vestibule was opened and electrode was inserted into scala vestibuli. ABI surgery was done through retrosigmoid approach. Foramen of Lushka was found to be closed. After careful dissection, lateral recess was identified and ABI electrode plate was successfully placed into lateral recess.

Two days after the surgery, her initial CI fitting was done. ABI was fitted 2 weeks after the surgery. In the following days her $\mathrm{ABI}$ was reprogrammed in two more sessions. As the patient resides abroad and she has chronic bone fracture problems, the follow-up fitting sessions are conducted at her local ABI center. According to her clinician's report, around 1 year after the surgery she responded to sound at $50 \mathrm{~dB}$ with her $\mathrm{CI}$ alone and with CI-ABI. But no responses were observed with the ABI alone. Moreover, she did not respond to tonal stimuli with either device or CI-ABI together. After around 2 years of simultaneous CI-ABI use she can respond to environmental sounds at $55 \mathrm{~dB}$ hearing level (HL) and at $60 \mathrm{~dB}$ HL with her CI and ABI alone respectively. When used bilaterally, responses are at $50 \mathrm{~dB}$ HL. With CI, she also had response to $500 \mathrm{~Hz}$ tonal stimuli, but no tonal stimuli related response was observed with ABI. She has five active electrodes for ABI. Others are disabled either due to absence of responses to individual electrode stimulation or to side effects.

Family reported positive impressions about the progress with both devices. They have reported that she started to respond to sounds with her CI at about 9 months after the surgery. Moreover, it was reported that she can produce some vocalization, and use signing for pointing out the musical instruments.

The initial progress was quite good in terms of responses to environmental sounds, further progress was also noticed in a recent test. At 3.5 years' postop, she responds to pure tones between 500 and $4000 \mathrm{~Hz}$ at between 40 to $55 \mathrm{~dB}$ with CI and at between 50 to $65 \mathrm{~dB}$ with $\mathrm{ABI}$ (Fig. 3A). With both devices she can respond to pure tones at between 40 and $45 \mathrm{~dB}$ (Fig. 3B) and to speech sounds at $35 \mathrm{~dB}$.

In case of CHARGE syndrome, the assessment of unilateral CI can make ABI decision difficult. Since ABI was implanted at the initial surgery, it took away any question about whether an ABI should be introduced earlier or later in the patient's course. Since acquisition of sound detection and more complex auditory information takes longer in these children, the delay between $\mathrm{CI}$ and subsequent $\mathrm{ABI}$ may have been considerable.

\section{Case 2}

The case was diagnosed after he failed at new born screening. In his first $\mathrm{ABR}$ testing with $90 \mathrm{~dB}$ HL click stimulus no wave $\mathrm{V}$ was observed, but cochlear microphonics were observed at both ears with stimulus polarity change. He was then fitted with conventional hearing aids but due to suboptimal progress with hearing aids, CI was considered. Preop insert ear phone testing results obtained with pure tones for right ear are shown in Figure 4, no response was observed at left ear.

HRCT showed normal cochlea and vestibular structures but bilateral cochlear aperture hypoplasia. MRI findings confirmed
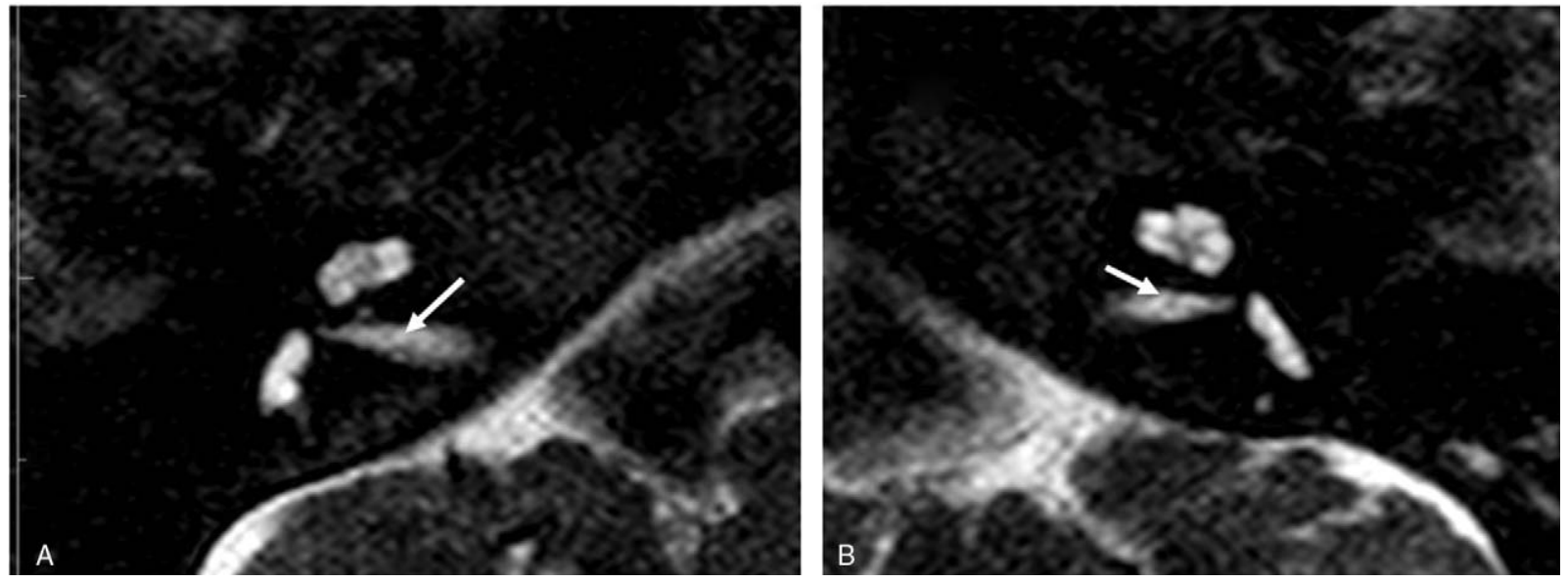

FIG. 2. Bilateral severe cochlear nerve deficiency as observed on MRI. MRI indicates magnetic resonance imaging. 


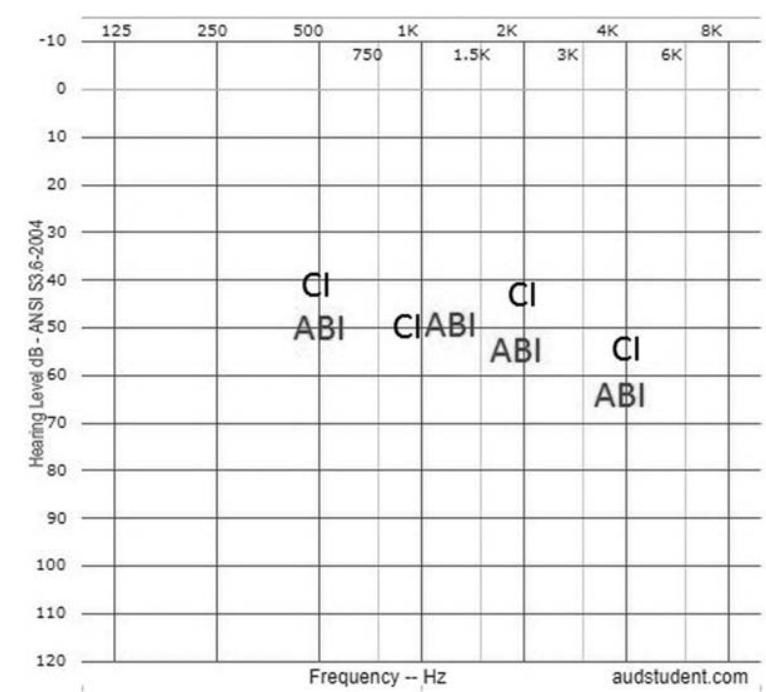

A

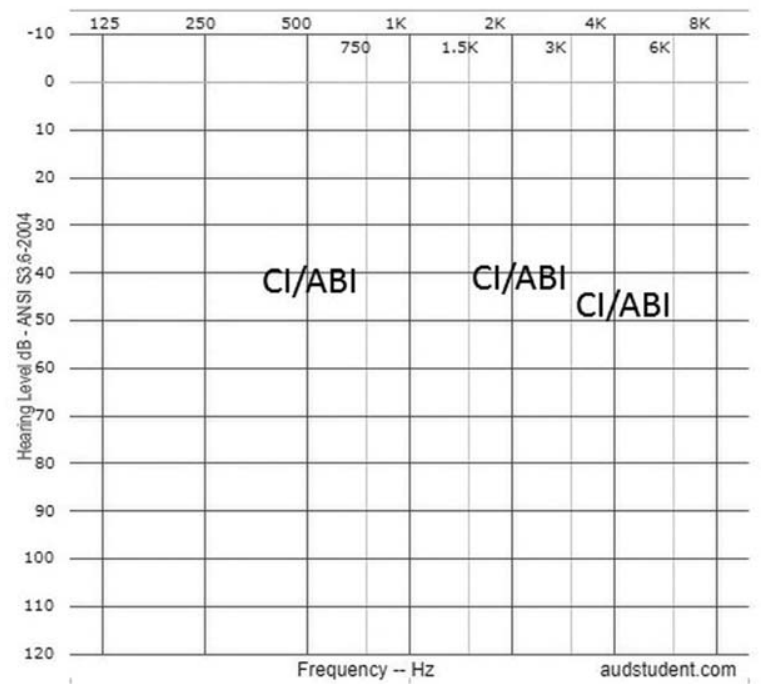

B

FIG. 3. Aided responses to pure tones with each device alone $(A)$, and both $(B)$.

a hypoplastic $\mathrm{CN}$ on the right ear and $\mathrm{CN}$ aplasia on left. In this particular case, there was no CI indication on the left side and based on radiological and audiological findings, CI on right, ABI on left was planned. The surgery was done when he was 2 years and 6 months.

CI surgery was uneventful. ABI electrode was placed successfully into lateral recess. CI was fitted 2 days after the surgery and ABI was fitted 1 month after the surgery. Around 2 to 3 months after the initial fitting for ABI, he started to respond to sounds at 50 to $60 \mathrm{~dB} \mathrm{HL}$ with each device. At around 1.5 years after the initial fittings the aided thresholds are far better, around 20 to $30 \mathrm{~dB}$ with each device and bilaterally when tested with speech sounds (Supplemental Content 2, http://links.lww.com/MAO/A938).

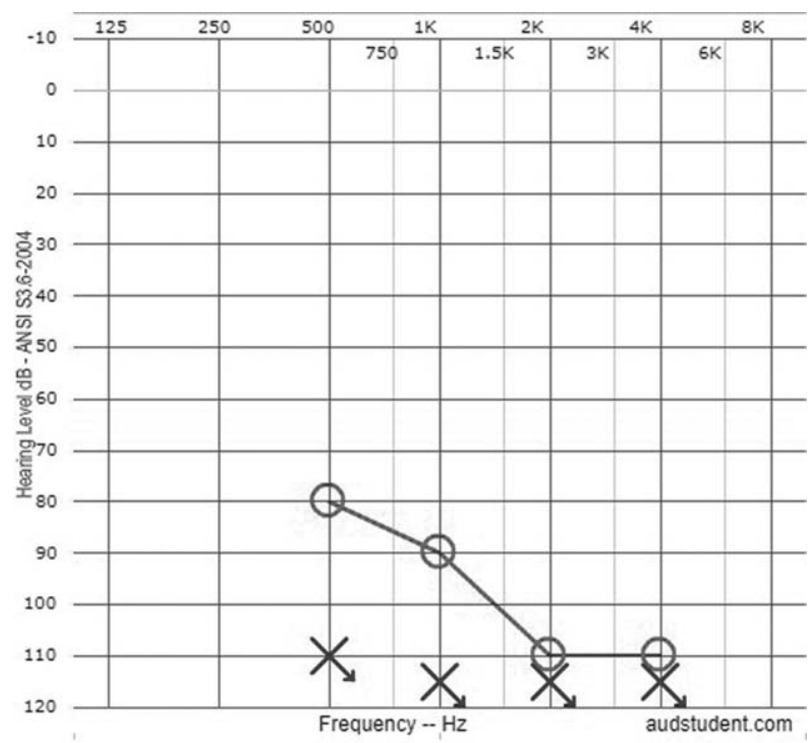

FIG. 4. Insert ear phone test results on right ear. No response was observed on left ear.

\section{Case 3}

1 year 9 months old female patient was referred for ABI. Preop insert earphone testing is given in Figure 5. No wave V could be observed at both ears with click stimulus at $90 \mathrm{dH} \mathrm{HL}$. Tympanometric tests revealed normal results.

HRCT and MRI demonstrated bilateral common cavity and presence of right $\mathrm{CVN}$, but no CVN was observed on the left side. CI was contraindicated on the left side and based on radiological and audiological findings, $\mathrm{CI}$ on right and $\mathrm{ABI}$ on left was planned. Surgery was done when she was 1 year 9 months old in June 2016.

CI surgery was done via transmastoid labryinthotomy approach on the right side. Intraoperative x-ray showed a straight electrode and therefore double labyrinthotomy

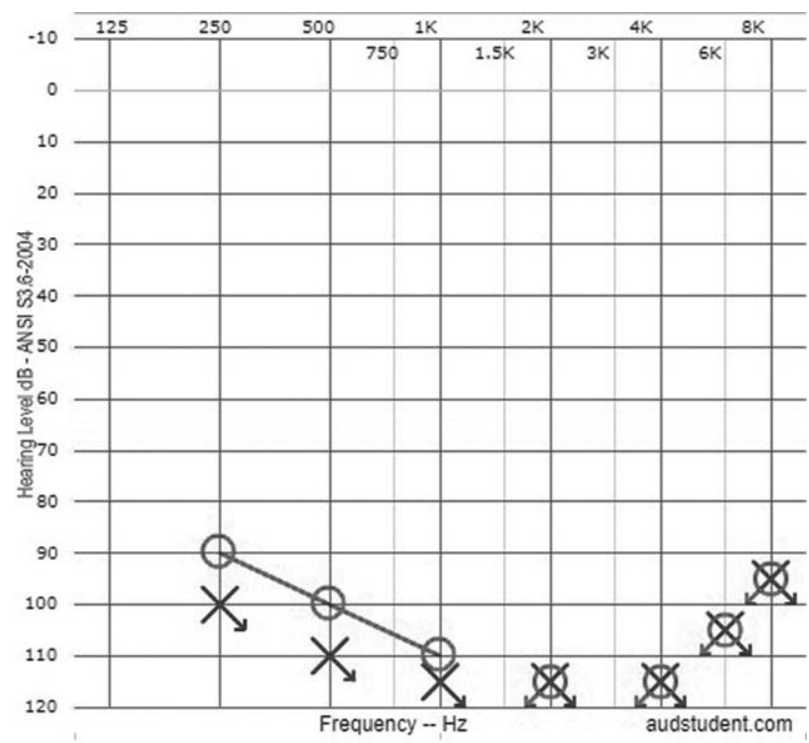

FIG. 5. Insert ear phone test results. No response was observed on left ear. 


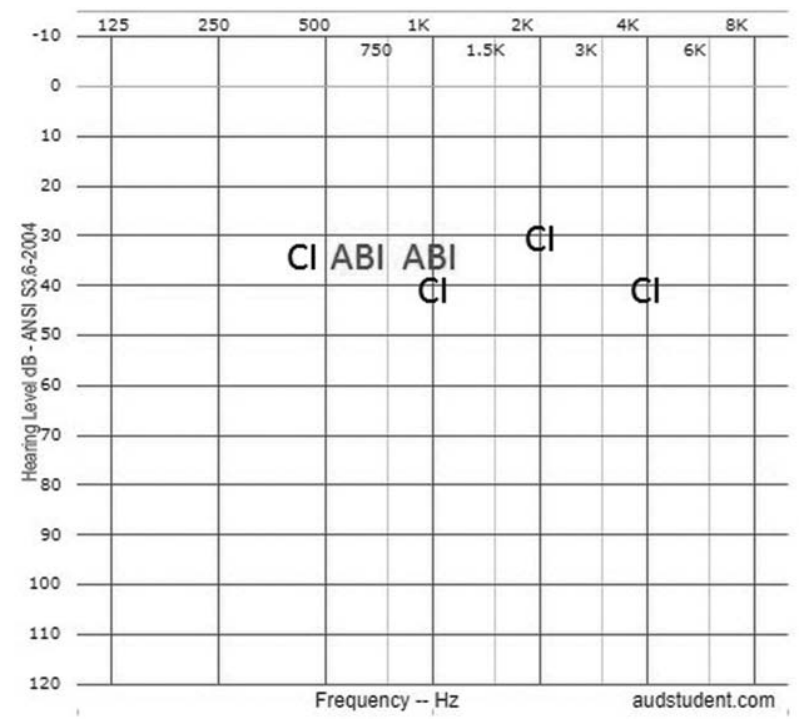

FIG. 6. Aided gain testing results 9 months after the surgery.

approach was used which resulted in an electrode position circling around the common cavity. Then ABI was placed via retrosigmoid approach on the left side, uneventfully into lateral recess. Her CI was fitted 2 days and ABI fitted 3 weeks after the surgery. At around 9 months after the surgery her aided thresholds are within daily conversation limits with each device alone tested through pure tones (Fig. 6). When bilaterally fitted, aided thresholds tested with speech sounds $/ \mathrm{ba} / \mathrm{ans} / \mathrm{sh} /$ were at $35 \mathrm{~dB}$ and 40 to $45 \mathrm{~dB}$ respectively.

\section{DISCUSSION}

Multiple studies showed that implantation within first few years of life brings superior outcomes compared with implantation in following years (13-15). In fact, issue of interest in our current work is providing bilateral hearing to pediatric patients with severe inner ear malformations as soon as possible. Our treatment option in cases with bilateral hypoplastic $\mathrm{CN}$ or bilateral severely hypoplastic $\mathrm{CN}$ along with observable hearing thresholds during insert ear testing is bilateral CI application. If one side has definite $\mathrm{ABI}$ indication such as cochlear nerve aplasia, with cochlear nerve deficiency on the contralateral side as in the three cases presented here, a more radical approach was chosen as the outcomes of implantation were foreseen to be suboptimal due to $\mathrm{CN}$ or $\mathrm{CVN}$ deficiency. Although they were referred for ABI, observing auditory thresholds unilaterally with insert ear phones made them candidates for CI in spite of hardly visible $\mathrm{CN}$. Their contralateral ears had absent $\mathrm{CN}$ or $\mathrm{CVN}$ making them already a candidate for ABI. Bilateral CI to be replaced in future with an $\mathrm{ABI}$ in case of insufficient progress, might have been another option but progress monitorization with bilateral CI and scheduling another surgery for ABI may take time. In 15 similar cases we performed $\mathrm{CI}$ implantation first and contralateral ABI later. Monitoring the progress of CI patients with $\mathrm{CN}$ deficiency and subsequently performing $\mathrm{ABI}$ can take around 1 to 2 years or more. If the initial CI surgery can be done around 7 to 8 months of age, and ABI decision and surgery is done at 1.5 years, this can provide bilateral stimulation within an acceptable time frame. However, it is not uncommon to have patients who apply for surgery around the age of two due to delays in diagnosis. Important time for development may be lost with this algorithm. As a result, we started to perform simultaneous $\mathrm{CI}$ and $\mathrm{ABI}$ implantation to avoid delay in hearing stimulation.

Our findings in terms of aided thresholds are promising as two patients showed responses to sounds within conversational limits in a relatively short period after the initial fittings. For one patient progress in terms of audition was slower compared with others, possibly due to comorbid CHARGE syndrome. In fact, existence of comorbid disorders has been found to result in suboptimal progress in higher level auditory and language skills among pediatric ABI users (8). Actually, the importance of bilateral application is prominently observed in this case, as clear responses to sounds through both devices were observed around 2 years after the surgery for this patient. If only CI or ABI had been applied, the outcomes may have been worse in this case. Especially for this case, and also for the others as well, our audiological outcomes in terms of aided thresholds show that children are easily adapted to bimodal stimulation through $\mathrm{CI}$ and $\mathrm{ABI}$. One may argue that aided thresholds may not be a very reliable proof for this statement, but it is observed that all bimodal users respond to speech sounds within conversational limits when both devices are working.

Choosing the better side for CI surgery is important. In our $\mathrm{CI}$ and $\mathrm{ABI}$ program, we have not observed any patient who did not respond to sounds after ABI application despite severe IEMs. Therefore, it can be concluded that inner ear development and cochlear nucleus development are not definitely correlated and the worst side in terms of anatomy can be chosen for the ABI. For CI, side with a response with insert ear phone test, or more developed cochlea or $\mathrm{CN}$ is preferred. Therefore, decision is made not only with MRI, but with audiological and MRI findings.

CI surgery may be complicated in some of these cases with complex inner ear anatomy. Case 1 had a facial nerve anomaly necessitating opening the vestibule and inserting the electrode into scala vestibuli. Second case had electrode migration into IAC which necessitated a repositioning of the electrode via double labyrinthotomy approach. Therefore, these cases may have prolonged surgery time. However, in centers who have experience in CI surgery in complex IEMs and ABI, it is feasible to perform simultaneous approach.

One important point here is that this procedure is not done in all cases of hypoplastic CN. Here, we propose that if $\mathrm{CN}$ is demonstrable on MRI having a size of at least $50 \%$ of ipsilateral $\mathrm{FN}$, it is advisable to perform unilateral or bilateral CI. ABI is done if an extremely thin and difficult to visualize $\mathrm{CN}$ is present. If this is the case, 
then no time will be lost with $\mathrm{CI}$ in such an ear. At this point we suggest the following algorithm for pediatric patients with complex inner ear malformations who are candidates of $\mathrm{CI}$ and/or $\mathrm{ABI}$; if there is no observable $\mathrm{CN}$ bilaterally on radiological imaging, bilateral $\mathrm{ABI}$ is indicated. In case of no $\mathrm{CN}$ at one side, but a $\mathrm{CN}$ on the contralateral side, which is at least $50 \%$ of the size of ipsilateral normal FN, application of a CI first at around the age of 8 months, then a CI or an ABI to the contralateral ear at around the age of 1 is suggested. Lastly, if there is no visible $\mathrm{CN}$ on one side, but a hardly visible $\mathrm{CN}$ on the other side simultaneous CI-ABI application may be considered. This application should be the choice especially when the candidate is evaluated at a later age, and when there is a comorbid disorder in addition to hearing loss. Schematic for this algorithm is given in Supplemental Content 3, http://links. lww.com/MAO/A939.

Based on the experience in the current work, we can say that if CI is successful, the child will receive the benefit of bimodal stimulation as soon as possible. On the other hand, if the CI is not successful, then the child will not lose the valuable time for ABI application; as ABI will have already started providing stimulation.

Although combination of CI and ABI surgery carries audiological advantages to children, the procedure involves more surgical risks necessitating very experienced surgical team. Surgeon is faced with a hypoplastic cochlea where it is difficult to locate the cochlea with abnormal facial nerve course, complicating the procedure. If the center is inexperienced in complex CI surgery, duration of anesthesiology before $\mathrm{ABI}$ surgery is increased. ABI surgery needs to be completed also within 3 hours so that the child will have maximum 5 to 6 hours of anesthesia. Blood and fluid loss should be kept to a minimum. Therefore, experienced pediatric anesthesiology is of vital importance. Advantage is that two complex procedures are managed in one session and bilateral hearing sensation is provided 1 month after surgery resolving the issue of providing hearing as soon as possible. However, it is important to perform this procedure in centers who are very experienced in CI surgery in complex malformations and pediatric neurosurgery. Keeping in mind these concerns a less radical approach may be application of ABI first, and CI later within 1 month. In this case scheduling of the surgeries will be very important. Also, the child will have to have two surgical sessions, which may be distressing for patient and parents.

Acknowledgments: Audiograms in this article were generated with AudGen v0.8 (http://audsim.com/audgen/AudGen.html).

\section{REFERENCES}

1. Young NM, Kim FM, Ryan ME, et al. Pediatric cochlear implantation of children with eight nerve deficiency. Int J Pediatr Otorhinolaryngol 2012;76:1442-8.

2. Vincenti V, Ormitti F, Ventura E, et al. Cochlear implantation in children with cochlear nerve deficiency. Int J Pediatr Otorhinolaryngol 2014;78:912-7.

3. Zhang Z, Li Y, Hu L, et al. Cochlear implantation in children with cochlear nerve deficiency: A report of nine cases. Int $J$ Pediatr Otorhinolaryngol 2012;76:1188-95.

4. Buchman C, Teagle HFB, Roush PA, et al. Cochlear implantation in children with labyrinthine anomalies and cochlear nerve deficiency: Implications for auditory brainstem implantation. Laryngoscope 2011;121:1979-88.

5. Oker N, Loundon N, Marlin S, et al. Bilateral implantation in children with cochleovestibular nerve hypoplasia. Int $J$ Pediatr Otorhinolaryngol 2009;73:1470-3.

6. Colletti L, Wilkinson EP, Colletti V. Auditory brainstem implantation after unsuccessful cochlear implantation of children with diagnosis of cochlear nerve deficiency. Ann Otol Rhinol Laryngol 2013;122:605-12.

7. Sennaroğlu L, Sennaroğlu G, Yücel E, et al. Long-term results of $\mathrm{ABI}$ in children with severe inner ear malformations. Otol Neurotol 2016;37:865-72.

8. Yücel E, Aslan F, Özkan HB, et al. Recent rehabilitation experience with pediatric ABI users. $J$ Int Adv Otol 2015;11:110-3.

9. Colletti L, Colletti G, Mandalà M, et al. The therapeutic dilemma of cochlear nerve deficiency: Cochlear or brainstem implantation? Otol Neurotol 2014;151:308-14.

10. Sennaroğlu L, Colletti V, Lenarz T, et al. Consensus statement: Long-term results of $\mathrm{ABI}$ in children with complex inner ear malformations and decision making between $\mathrm{CI}$ and $\mathrm{ABI}$. Cochlear Implants Int 2016;17:163-71.

11. Sennaroğlu G, Cicek-cinar B, Batuk M, et al. Bimodal stimulation: One side cochlear implant and contralateral auditory brainstem implant. Presented at the 12th European Symposium on Pediatric Cochlear Implantation, Touluse, June 18-21, 2015.

12. Sennaroğlu L, Bajin MD. Classification and current management of inner ear malformations. Balkan Med 2017;34:397-411.

13. Kirk KI, Miyamoto RT, Lento CL, et al. Effects of age at implantation in young children. Ann Otol Rhinol Laryngol 2002;111: $69-73$.

14. Kirk KI, Miyamoto RT, Ying E, et al. Cochlear implantation in young children: Effects of age at implantation and communication mode. Volta Rev 2000;102:127-44.

15. Houston DM, Miyamoto RT. Effects of early auditory experience on word learning and speech perception in deaf children with cochlear implants: Implications for sensitive periods of language development. Otol Neurotol 2010;31:1248-53. 